\title{
Research Article \\ Efficient MPEG-2 to H.264/AVC Transcoding of Intra-Coded Video
}

\author{
Jun Xin, ${ }^{1}$ Anthony Vetro, ${ }^{2}$ Huifang Sun, ${ }^{2}$ and Yeping $\mathrm{Su}^{3}$ \\ ${ }^{1}$ Xilient Inc., 10181 Bubb Road, Cupertino, CA 95014, USA \\ ${ }^{2}$ Mitsubishi Electric Research Labs, 201 Broadway, Cambridge, MA 02139, USA \\ ${ }^{3}$ Sharp Labs of America, 5750 NW Pacific Rim Boulevard, Camas, WA 98607, USA
}

Received 3 October 2006; Revised 30 January 2007; Accepted 25 March 2007

Recommended by Yap-Peng Tan

This paper presents an efficient transform-domain architecture and corresponding mode decision algorithms for transcoding intra-coded video from MPEG-2 to H.264/AVC. Low complexity is achieved in several ways. First, our architecture employs direct conversion of the transform coefficients, which eliminates the need for the inverse discrete cosine transform (DCT) and forward H.264/AVC transform. Then, within this transform-domain architecture, we perform macroblock-based mode decisions based on H.264/AVC transform coefficients, which is possible using a novel method of calculating distortion in the transform domain. The proposed method for distortion calculation could be used to make rate-distortion optimized mode decisions with lower complexity. Compared to the pixel-domain architecture with rate-distortion optimized mode decision, simulation results show that there is a negligible loss in quality incurred by the direct conversion of transform coefficients and the proposed transformdomain mode decision algorithms, while complexity is significantly reduced. To further reduce the complexity, we also propose two fast mode decision algorithms. The first algorithm ranks modes based on a simple cost function in the transform domain, then computes the rate-distortion optimal mode from a reduced set of ranked modes. The second algorithm exploits temporal correlations in the mode decision between temporally adjacent frames. Simulation results show that these algorithms provide additional computational savings over the proposed transform-domain architecture while maintaining virtually the same coding efficiency.

Copyright (C) 2007 Jun Xin et al. This is an open access article distributed under the Creative Commons Attribution License, which permits unrestricted use, distribution, and reproduction in any medium, provided the original work is properly cited.

\section{INTRODUCTION}

The latest video compression standard, known as H.264/AVC [1], is able to achieve significantly improved compression efficiency over prior standards such as MPEG-2. Due to its superior performance, it is being widely adopted for a broad range of applications, including broadcasting, consumer electronics storage, surveillance, video conference and mobile video. As H.264/AVC becomes more widely deployed, the number of devices that are capable of decoding H.264/AVC bitstreams will grow. In fact, multiformat standard decoder solutions, which have the capability to decode multiple video compression formats including MPEG2, H.264/AVC, and VC-1, are becoming available. This will give those in the content delivery chain greater flexibility in the format that video content is authored, edited, transmitted, and stored.

However, with the success of MPEG-2 in various application domains, there exists not only a significant amount of legacy content, but also equipment for producing MPEG-2 content and networking infrastructure to transmit this content. To minimize the need to upgrade all of this equipment at once and ease the transition to the new H.264/AVC coding format, there is a strong need for efficient transcoding from MPEG-2 to H.264/AVC. This topic has received much attention from the research community in recent years [2-4]. In this paper, we focus on a particular subset of this larger problem, which is the transcoding of intra-coded video.

Intra-only video coding is a widely used coding method in television studio broadcast, digital cinema, and surveillance video applications. The main reason is that intra-coded video is easier to edit than video with predictively coded frames. Prior experiments and demonstrations have shown that H.264/AVC intra-coding has an excellent performance, even compared to state-of-the-art still image coding schemes such as JPEG 2000 [5]. As an acknowledgment of such needs, JVT is currently working on an intra-only profiles, which will 
include tools for coding of $4: 4: 4$ sampled video and possibly lower $4: 2: 0$ sampling formats as well [6].

The primary aim of the transcoder in this paper is to provide more efficient network transmission and storage. A conventional method of transcoding MPEG-2 intra-coded video to H.264/AVC format is shown in Figure 1. In this architecture, the transcoder first decodes an input MPEG-2 video to reconstruct the image pixels, and then encodes the pixels in a frame in the H.264/AVC format. We refer to this architecture as a pixel-domain transcoder (PDT).

It is well known that transform-domain techniques may be simpler since they eliminate the need of inverse transform and forward transform operations. However, in the case of MPEG-2 to H.264/AVC transcoding, the transform-domain approach must efficiently solve the following two problems. The first problem is a transform mismatch, which arises from the fact that MPEG-2 uses a DCT, while H.264/AVC uses a low-complexity integer transform, hereinafter referred to as HT. Therefore, an efficient algorithm for DCT-to-HT coefficient conversion that is simpler than the trivial concatenation of IDCT and HT is needed. A number of algorithms that perform this conversion have been recently reported in the literature [7-9]. Since this conversion is an important component of the transform-domain architecture, we briefly describe our previous work [7] and provide a comparison to other works in this paper. The second problem with a transform-domain architecture is in the mode decision. In H.264/AVC, significant coding efficiency gains are achieved through a wide variety of prediction modes. To achieve the best coding efficiency, the rate and distortion for each coding mode are calculated, then the optimal mode is determined. In conventional architectures, the distortion is calculated based on original and reconstructed pixels with a candidate mode. However, in the proposed transform-domain architecture, this distortion is calculated based on the transform coefficients yielded from each candidate mode.

Figure 2 illustrates our proposed transform-domain transcoder, in which the primary areas of focus are highlighted. In Section 2, we will describe what we refer to as the S-transform, or the DCT-to-HT conversion of transform coefficients. Its integer implementation is also discussed. Then, in Section 3, we present the architecture for performing a rate-distortion optimized mode decision in the transform domain, including a novel means of calculating distortion in the transform domain. It is noted that the most time consuming operation in the transcoder is the mode decision process, which determines the particular method for predictively coding macroblocks. Section 4 describes a two fast mode decision algorithms that achieve further speedup. Simulation results that validate the efficiency of the various processes and fast mode decision algorithms are discussed in Section 5. Finally, we provide a summary of our contributions and some concluding remarks in Section 6.

\section{EFFICIENT DCT-TO-HT CONVERSION}

This section summarizes the key elements of our prior work on direct conversion of transform coefficients [7]. We present the transform matrix itself, review the fast implementation and study the impact of integer approximations. We also discuss some of the related works in this area that have been recently published.

\subsection{Transformation matrix}

As a point of reference, Figure 3(a) shows a pixel-domain implementation of the DCT-to-HT conversion. The input is an $8 \times 8$ block $(X)$ of DCT coefficients. An inverse DCT (IDCT) is applied to $X$ to recover an $8 \times 8$ pixel block $(x)$. The $8 \times 8$ pixel block is divided evenly into four $4 \times 4$ blocks $\left(x_{1}, x_{2}, x_{3}, x_{4}\right)$. Each of the four blocks is passed to a corresponding HT to generate four $4 \times 4$ blocks of transform coefficients $\left(Y_{1}, Y_{2}, Y_{3}, Y_{4}\right)$. The four blocks of transform coefficients are combined to form a single $8 \times 8$ block $(Y)$. This is repeated for all blocks of the video.

Figure 3(b) illustrates the direct conversion of transform coefficients, which we refer to as the S-transform. Let $X$ denote an $8 \times 8$ block of DCT coefficients, the corresponding HT coefficient block $Y$, consisting of four $4 \times 4$ HT blocks, is given by

$$
Y=S * X * S^{T}
$$

As derived in [7], the kernel matrix $S$ is

$$
S=\left(\begin{array}{cccccccc}
a & b & 0 & -c & 0 & d & 0 & -e \\
0 & f & g & h & 0 & -i & -j & k \\
0 & -l & 0 & m & a & n & 0 & -o \\
0 & p & j & -q & 0 & r & g & s \\
a & -b & 0 & c & 0 & -d & 0 & e \\
0 & f & -g & h & 0 & -i & j & k \\
0 & l & 0 & -m & a & -n & 0 & o \\
0 & p & -j & -q & 0 & r & -g & s
\end{array}\right)
$$

where the values $a \cdots s$ are (rounded off to four decimal places)

$$
\begin{array}{rrrr}
a=1.4142, & b=1.2815, & c=0.45, & d=0.3007, \\
e=0.2549, & f=0.9236, & g=2.2304, & h=1.7799, \\
i=0.8638, & j=0.1585, & k=0.4824, & l=0.1056, \\
m=0.7259, & n=1.0864, & o=0.5308, & p=0.1169, \\
q=0.0922, \quad r=1.0379, \quad s=1.975 .
\end{array}
$$

\subsection{Fast conversion}

The symmetry of the kernel matrix can be utilized to design fast implementations of the transform. As suggested by (1), the 2D S-transform is separable. Therefore, it can be achieved through 1D transforms. Hence, we will describe only the computation of the 1D transform.

Let $\vec{z}$ be an 8 -point column vector, and a vector $\vec{Z}$ the $1 \mathrm{D}$ transform of $\vec{z}$. The following steps provide a method to 


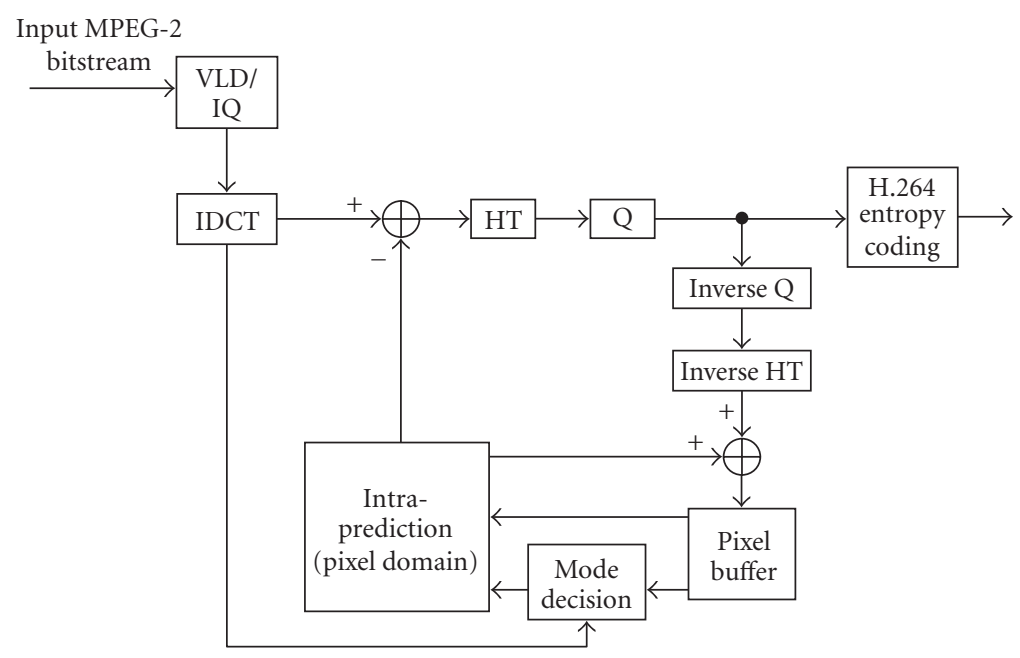

VLD: variable-length decoding

(I)Q: (inverse) quantization

IDCT: inverse discrete cosine transform

HT: H.264/AVC $4 \times 4$ transform

FIgURE 1: Pixel-domain intra-transcoding architecture.

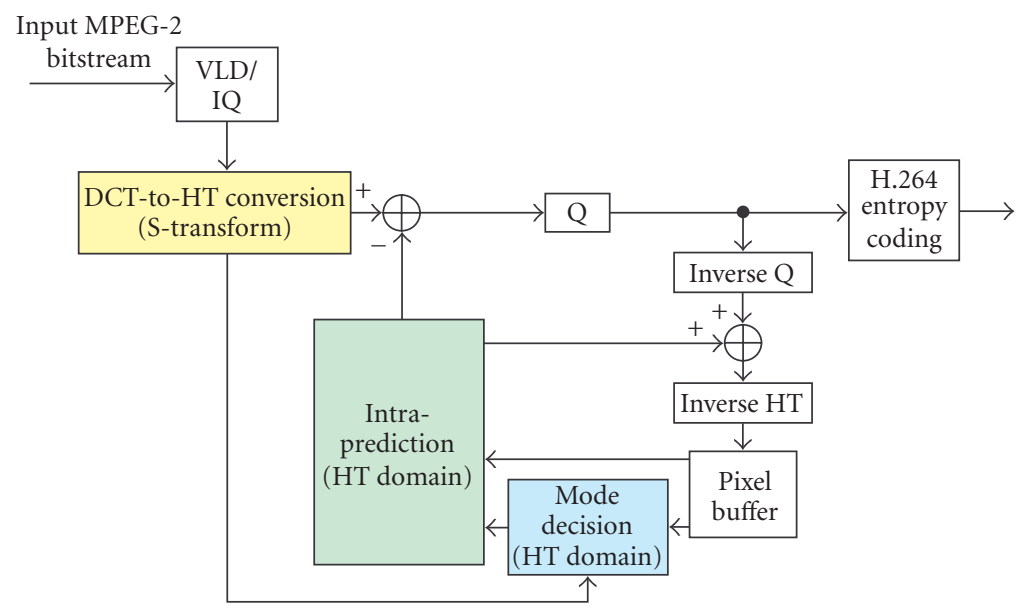

VLD: variable-length decoding

(I)Q: (inverse) quantization

IDCT: inverse discrete cosine transform

HT: H.264/AVC $4 \times 4$ transform

FIGURE 2: Transform-domain intra-transcoding architecture.

determine $\vec{Z}$ efficiently from $\vec{z}$, which is also shown in Figure 4 as a flow graph,

$$
\begin{aligned}
& m 1=a \times z[1], \\
& m 2=b \times z[2]-c \times z[4]+d \times z[6]-e \times z[8], \\
& m 3=g \times z[3]-j \times z[7], \\
& m 4=f \times z[2]+h \times z[4]-i \times z[6]+k \times z[8], \\
& m 5=a \times z[5], \\
& m 6=-l \times z[2]+m \times z[4]+n \times z[6]-o \times z[8], \\
& m 7=j \times z[3]+g \times z[7],
\end{aligned}
$$

$$
\begin{aligned}
m 8 & =p \times z[2]-q \times z[4]+r \times z[6]+s \times z[8] \\
Z[1] & =m 1+m 2, \\
Z[2] & =m 3+m 4, \\
Z[3] & =m 5+m 6 \\
Z[4] & =m 7+m 8 \\
Z[5] & =m 1-m 2, \\
Z[6] & =m 4-m 3, \\
Z[7] & =m 5-m 6 \\
Z[8] & =m 8-m 7
\end{aligned}
$$




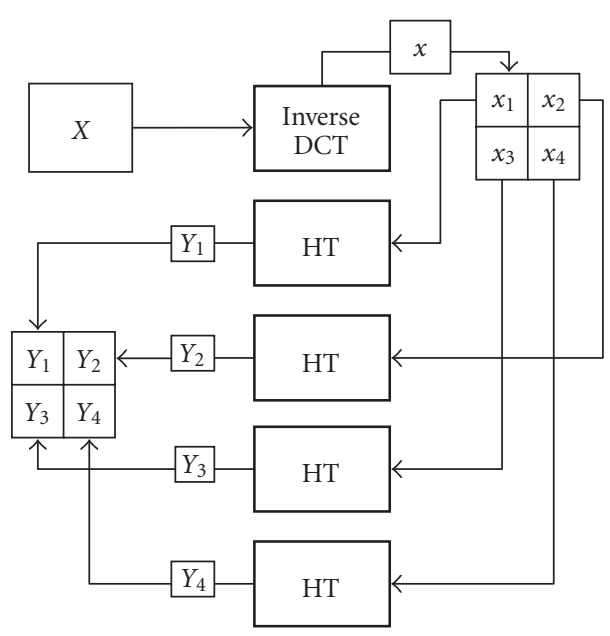

(a)

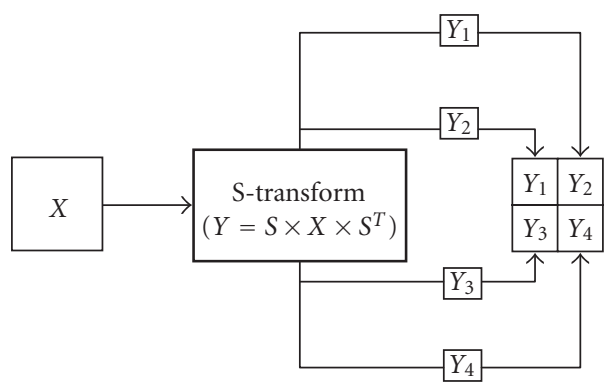

(b)

FIgure 3: Comparison between two DCT-to-HT conversion schemes: (a) pixel domain, (b) transform domain.

This method requires 22 multiplications and 22 additions. It follows that the 2 D S-transform needs $352(=16 \times 22)$ multiplications and 352 additions, for a total of 704 operations.

The pixel-domain implementation includes one IDCT and four HT operations. Chen's fast IDCT implementation [10], which we refer to as the reference IDCT, needs 256(= $16 \times 16)$ multiplications and $416(=16 \times 26)$ additions. Each HT needs $16(=2 \times 8)$ shifts and $64(=8 \times 8)$ additions [11]. The four HT then need 64 shifts and 256 additions. It follows that the overall computational requirement of the pixeldomain processing is 256 multiplications, 64 shifts, and 672 additions, for a total of 992 operations.

Thus, the fast S-transform saves about 30\% of the operations when compared to the pixel-domain implementation. In addition, the S-transform can be implemented in just two stages, whereas the conventional pixel-domain processing using the reference IDCT requires six stages (four for the reference IDCT and two for the HT). In the following subsection, an integer approximation of the S-transform is described.

\subsection{Integer approximation}

Floating-point operations are generally more expensive to implement than integer operations, so we also study the inte-

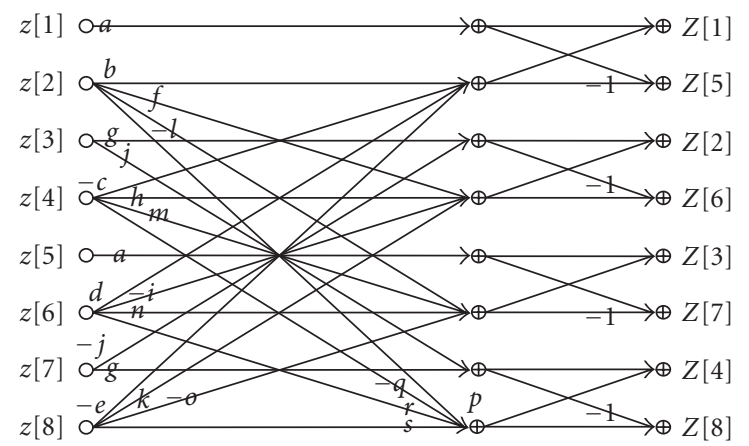

FIGURE 4: Fast algorithm for the transform-domain DCT-to-HT conversion.

ger approximation of the S-transform. To achieve an integer representation, we multiply $S$ by an integer that is a power of two, and use the integer transform kernel matrix to perform the transform using an integer-arithmetic. Then, the resulting coefficients are scaled down by proper shifting. In video transcoding applications, the shifting operations can be absorbed in the quantization. Therefore, no additional operations are required to use integer arithmetic.

Larger integers will generally lead to better accuracy. Typically, the number is limited by the microprocessor on which the transcoding is performed. We assume that most processors are capable of 32-bit arithmetic, so select a number that would satisfy this constraint. However, approximations for other processor constraints could also be determined.

The input DCT coefficients to the S-transform lie in the range of -2048 to 2047 and require 12 bits. The maximum sum of absolute values in any row of $S$ is 6.44, therefore the maximum dynamic range gain for the 2D S-transform is $6.44^{2}=41.47$, which implies $\log _{2}(41.47)=5.4$ extra bits or 17.4 bits total to represent the final S-transform results. For 32-bit arithmetic, the scaling factor must be smaller than the square root of $2^{32-17.4}$, that is, 157.4. The maximum integer satisfying this condition while being a power of two is 128. Therefore, the integer transform kernel matrix is $S I=$ round $\{128 \times S\}$. Similar to $S, S I$ has the form (2), but with the values $a$ through $s$ changed to the following integers:

$$
\begin{aligned}
& a=181, \quad b=164, \quad c=58, \quad d=38, \quad e=33, \\
& f=118, \quad g=285, \quad h=228, \quad i=111, \quad j=20 \text {, } \\
& k=62, \quad l=14, \quad m=93, \quad n=139, \quad o=68 \text {, } \\
& p=15, \quad q=12, \quad r=133, \quad s=253 \text {. }
\end{aligned}
$$

It is noted that the fast algorithm derived in the previous subsection for the S-transform can be applied to the above transform since SI and $S$ have the same symmetric property. Also, results reported in [7] demonstrate that the integer Stransform yields slight gains on the order of $0.2 \mathrm{~dB}$ compared to the reference pixel-domain approach. This gain is achieved since the integer S-transform avoids the rounding operation 


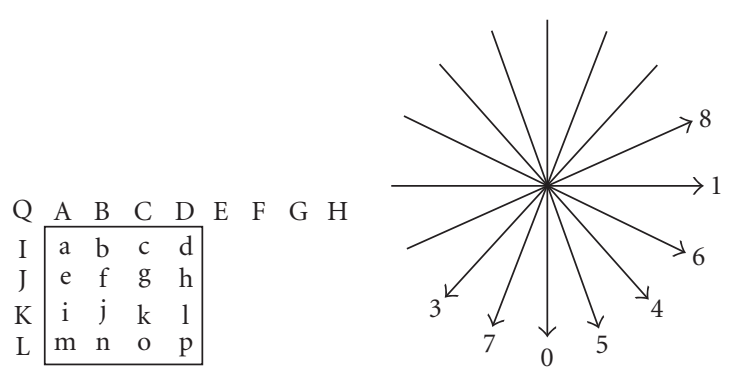

FIGURE 5: (a) Neighboring samples "A-Q" are used for prediction of samples “a-p." (b) Prediction mode directions (except DC_Pred).

after the IDCT and for intermediate values within the HT transform itself.

\subsection{Discussion}

The number of clock cycles required to execute different types of operations are machine dependent. In the above, it is assumed that integer addition, integer multiplication, and shifts consume the same number of clock cycles. However, to make the comparison more complete, let us assume that a multiplication needs 2 cycles and an addition/shift needs 1 cycle, which is the general case for TI C64 family DSP processors. The S-transform would then need 1056(352*2+352) cycles, while the conventional pixel-domain approach would need $1248(256 * 2+64+672)$ cycles. In addition, the above calculation has not taken into account that the reference IDCT needs floating point operations, which typically is more expensive than integer operations. Therefore, the proposed coefficient conversion is still more efficient.

Recently, there have been new algorithms developed for converting DCT coefficients to HT coefficients. One algorithm uses a factorized form of the $8 \times 8$ DCT kernel matrix [8]. Multiplications in the process of matrix multiplications are replaced by additions and shifts. However, this process introduces approximation errors and transcoding quality suffers. Following Shen's method, and taking advantage that the HT transform kernel matrix can be approximately decomposed to the $4 \times 4$ DCT transform kernel, a new algorithm was proposed in [9], where the conversion matrix is decomposed to sparse matrices. This algorithm is shown to be more efficient and more accurate than [8]. Although this approach has advantage in terms of computational complexity, it still has nontrivial approximation errors compared to our approach. More detailed comparison of the above algorithms could be found in [9]. Therefore, we believe that our proposed algorithm is preferred for high-quality applications.

\section{TRANSFORM-DOMAIN MODE DECISION ARCHITECTURE}

This section describes a transform-domain mode decision architecture, and presents a method of calculating distortion required for cost calculations in the mode decision process.

\subsection{Conventional mode decision}

Let us first consider the conventional H.264 pixel-domain mode decision (as implemented in the JM reference software), and in particular, the rate-distortion optimized (RDO) decision for the Intra_4 $\times 4$ modes. Figure 5(a) illustrates the candidate neighboring pixels "A-Q" used for prediction of current $4 \times 4$ block pixels "a-p." Figure 5(b) illustrates the eight directional prediction modes. In addition, DC prediction (DC_Pred) can also be used.

Consider the rate-distortion calculation in a video encoder with RDO_on, the conventional calculation of the Lagrange cost for one coding module (in this case for one $4 \times 4$ luma block) is shown in Figure 6. The prediction residual is transformed, quantized and entropy encoded to determine the rate, $R(m)$, for a given mode $m$. Then, inverse quantization and inverse transform are performed and then compensated with the prediction block to get the reconstructed signal. The distortion, denoted $\operatorname{SSD}_{\operatorname{REC}}(m)$, is computed as the sum of squared distance between the original block, $s$, and the reconstructed block, $\widetilde{s}(m)$ :

$$
\operatorname{SSD}_{\operatorname{REC}}(m)=\|s-\widetilde{s}(m)\|_{2}^{2},
$$

where $\|\cdot\|_{p}$ is the $L p$-norm. The Lagrange cost is computed using the rate and distortion as follows:

$$
\operatorname{Cost}_{4 \times 4}=\operatorname{SSD}_{\mathrm{REC}}(m)+\lambda_{M} * R(m),
$$

where $\lambda_{M}$ is the Lagrange multiplier, which may be calculated as a function of the quantization parameter. The optimal coding mode corresponds to the mode yielding the minimum cost.

Besides this RDO mode selection, a low-complexity algorithm, that is, with RDO_off, would only calculate the sum of absolute distance of the Hadamard-transformed prediction residual signal:

$$
\operatorname{SATD}(m)=\|T(s-\widehat{s}(m))\|_{1},
$$

where $\widehat{s}(m)$ is the prediction signal for the mode $m$. In this case, the cost function would then be given by

$$
\operatorname{Cost}_{4 \times 4}=\operatorname{SATD}(m)+\lambda_{M} * 4 *\left(1-\delta\left(m=m^{*}\right)\right),
$$

where $m^{*}$ is the most probable mode for the block.

\subsection{Transform-domain mode decision}

The proposed transform-domain mode decision calculates the Lagrange cost for each mode according to Figure 7, which is based on our previous work on H.264 encoding [12]. Compared to the pixel-domain approach, the transform-domain implementation has several major differences in terms of computation involved, which are discussed below.

First, the transform-domain approach saves one inverse HT computation for each candidate prediction mode. This is possible since the distortion is determined using the reconstructed and original residual HT coefficients. The details on this calculation are presented in the next subsection. 


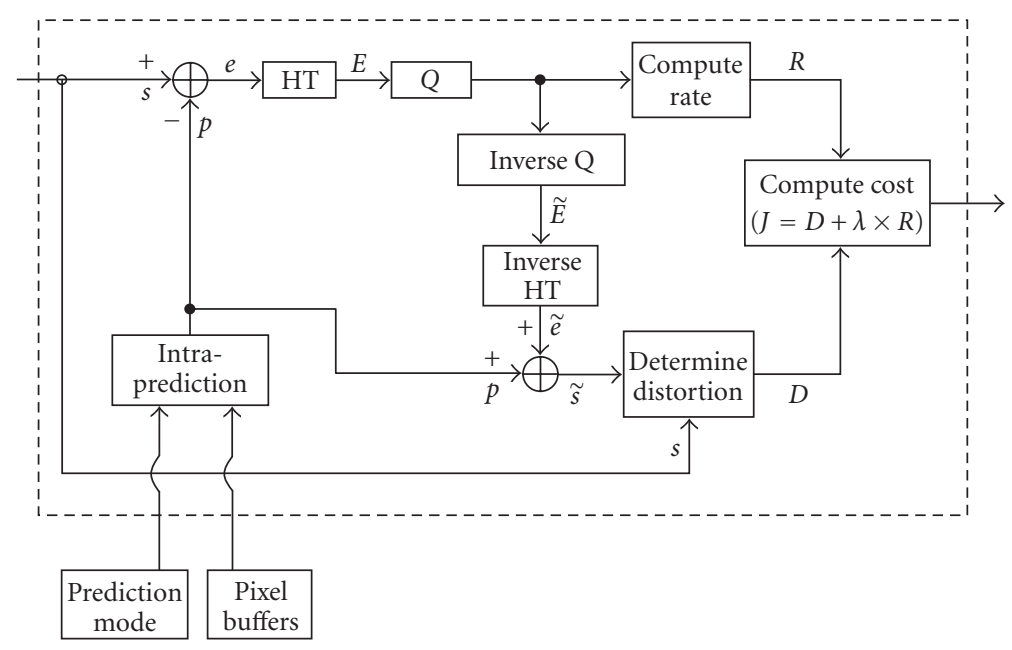

Figure 6: Pixel-domain RD cost calculation.

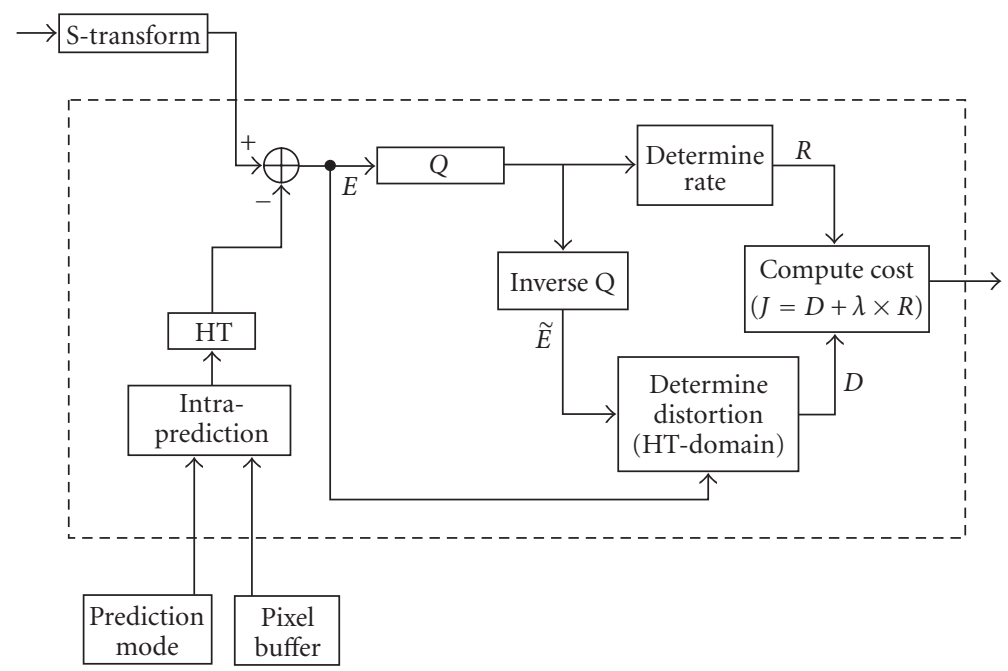

FIGURE 7: Transform-domain RD cost calculation.

Second, instead of operating on the prediction residual pixels, the HT now operates on the prediction signals. In [12], we have shown that the HT of some intra-prediction signals are very simple to compute. For example, there is only one nonzero DC element in the transformed prediction signal for DC_Pred mode. Therefore, additional computational saving are achieved.

\subsection{Distortion calculation in transform domain}

As described in the previous subsection and indicated in Figure 7, the distortion is calculated in the transform domain, or HT domain to be precise. Since the HT is not an orthonormal transform, it does not preserve the L2 norm (energy). However, the distortion can still be calculated with proper coefficient weighting [12].

Let $\tilde{s}=p+\tilde{e}$ denote the reconstructed signal, and let $s=p+e$ denote the original input signal, where $e$ and $\tilde{e}$ are the prediction residual error signal and the reconstructed residual signal, respectively, and $p$ is the prediction signal. The pixel-domain distortion, $\operatorname{SSD}_{\mathrm{REC}}(m)$, is given by (6). In the following, we derive the transform-domain distortion calculation.

First, we rewrite (6) in matrix form:

$$
D=\operatorname{trace}\left((s-\widetilde{s}) \times(s-\widetilde{s})^{T}\right),
$$

where $\widetilde{s}(m)$ is replaced with $\tilde{s}$ for simplicity. It follows that

$$
D=\operatorname{trace}\left((e-\tilde{e}) \times(e-\tilde{e})^{T}\right) .
$$

Let $E$ be the HT transformed residual signal and let $\widetilde{E}$ be the reconstructed HT transform coefficients through inverse scaling and inverse transform. We then have the following:

$$
\begin{gathered}
e=H^{-1} \times E \times\left(H^{T}\right)^{-1}, \\
\tilde{e}=\frac{\tilde{H}_{\mathrm{inv}} \times \widetilde{E} \times\left(\tilde{H}_{\mathrm{inv}}^{T}\right)}{64},
\end{gathered}
$$




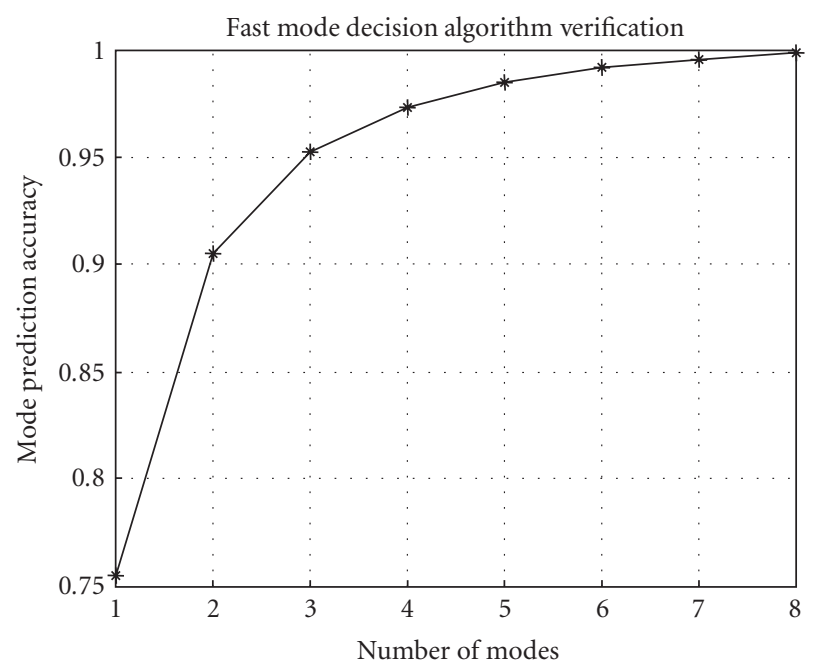

FIGURE 8: Number of test modes versus accuracy.

where $H$ and $\widetilde{H}_{\text {inv }}$ are the kernel matrices the forward HT transform and inverse HT transform used in the H.264/AVC decoding process, respectively, and are given by

$$
\begin{aligned}
H & =\left(\begin{array}{cccc}
1 & 1 & 1 & 1 \\
2 & 1 & -1 & -2 \\
1 & -1 & -1 & 1 \\
1 & -2 & 2 & -1
\end{array}\right), \\
\tilde{H}_{\text {inv }} & =\left(\begin{array}{cccc}
1 & 1 & 1 & \frac{1}{2} \\
1 & \frac{1}{2} & -1 & -1 \\
1 & -\frac{1}{2} & -1 & 1 \\
1 & -1 & 1 & -\frac{1}{2}
\end{array}\right) .
\end{aligned}
$$

Note that in (13), the scaling after inverse HT in the decoding process is already taken care of by the denominator 64 . It is easy to verify that

$$
\begin{gathered}
\tilde{H}_{\text {inv }}=H^{-1} \times M_{1}, \\
\tilde{H}_{\text {inv }}^{T}=M_{1} \times\left(H^{T}\right)^{-1},
\end{gathered}
$$

where $M_{1}=\operatorname{diag}(4,5,4,5)$. It follows from (12), (13), and (15) that

$$
\begin{aligned}
e-\tilde{e} & =H^{-1} \times E \times\left(H^{T}\right)^{-1}-\frac{\tilde{H}_{\text {inv }} \times \widetilde{E} \times\left(\tilde{H}_{\text {inv }}^{T}\right)}{64} \\
& =H^{-1} \times\left(E-\frac{M_{1} \times \widetilde{E} \times M_{1}}{64}\right) \times\left(H^{T}\right)^{-1} \\
& =H^{-1} \times\left(E-\widetilde{E} \otimes W_{1}\right) \times\left(H^{T}\right)^{-1},
\end{aligned}
$$

where $\otimes$ operator represents a scalar multiplication or entrywise multiplication, and $W_{1}$ is given by

$$
W_{1}=\frac{1}{64}\left(\begin{array}{llll}
16 & 20 & 16 & 20 \\
20 & 25 & 20 & 25 \\
16 & 20 & 16 & 20 \\
20 & 25 & 20 & 25
\end{array}\right)
$$

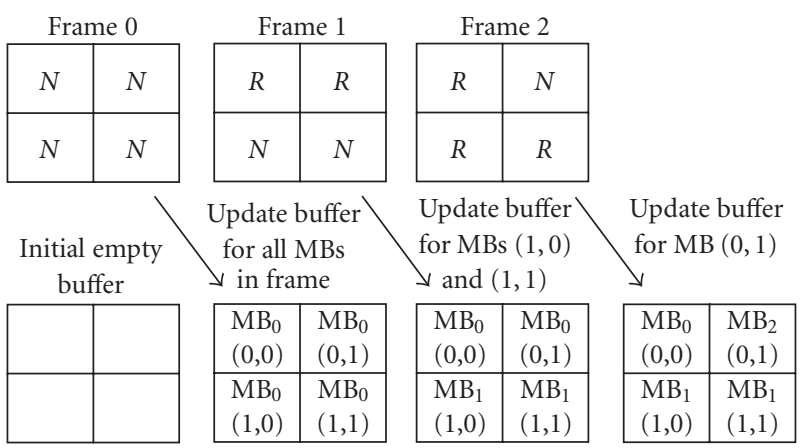

FIGURE 9: Example of buffer updating process used for mode decision based on temporal correlation. $N$ indicates that a new mode decision has been made, while $R$ indicates that the mode decision of the previously coded macroblock is reused.

Let $\Delta E=E-\widetilde{E} \otimes W_{1}$, and substituting (16) into (11) gives

$$
D=\operatorname{trace}\left(H^{-1} \times \Delta E \times\left(H^{T}\right)^{-1} \times H^{-1} \times \Delta E^{T} \times\left(H^{T}\right)^{-1}\right) .
$$

Denote $M_{2}=\left(H^{T}\right)^{-1} \times H^{-1}=\operatorname{diag}(0.25,0.1,0.25,0.1)$, we also have $\left(H^{T}\right)^{-1}=M_{2} \times H$, which then gives

$$
\begin{aligned}
D & =\operatorname{trace}\left(H^{-1} \times \Delta E \times M_{2} \times \Delta E^{T} \times M_{2} \times H\right) \\
& =\operatorname{trace}\left(\Delta E \times M_{2} \times \Delta E^{T} \times M_{2}\right)=\left\|\Delta E \otimes W_{2}\right\|_{2}^{2},
\end{aligned}
$$

where $W_{2}$ is given by

$$
W_{2}=\left(\begin{array}{cccc}
\frac{1}{4} & \frac{1}{\sqrt{40}} & \frac{1}{4} & \frac{1}{\sqrt{40}} \\
\frac{1}{\sqrt{40}} & \frac{1}{10} & \frac{1}{\sqrt{40}} & \frac{1}{10} \\
\frac{1}{4} & \frac{1}{\sqrt{40}} & \frac{1}{4} & \frac{1}{\sqrt{40}} \\
\frac{1}{\sqrt{40}} & \frac{1}{10} & \frac{1}{\sqrt{40}} & \frac{1}{10}
\end{array}\right) .
$$

Expanding $\Delta E$ gives the final forms of the transform-domain distortion:

$$
D_{\mathrm{HT}}(m)=\left\|\left(E-\widetilde{E}(m) \otimes W_{1}\right) \otimes W_{2}\right\|_{2}^{2} .
$$

Thus far, we have shown that with weighting matrices $W_{1}$ and $W_{2}$ to compensate for the different norms of HT, inverse HT and H.264/AVC quantization design, we can calculate the SSD distortion in the HT domain using (21).

In what follows, we analyze the computational complexity of the proposed distortion calculation. All following discussions are based on a $4 \times 4$ block basis. In (21), to avoid floating point operation in computing $\left(\widetilde{E}(m) \otimes W_{1}\right)$, we take the 1/64 constant out of the $L 2$-norm operator to yield

$$
D_{\mathrm{HT}}(m)=\frac{1}{64^{2}}\left\|\left(64 * E-\widetilde{E}(m) \otimes\left(W I_{1}\right)\right) \otimes W_{2}\right\|_{2}^{2},
$$


where $W I_{1}=64 * W_{1}$ is now an integer matrix. Let $Y=$ $64 * E-\widetilde{E}(m) \otimes W I_{1}$, and substituting $W_{2}$ into (22) gives

$$
\begin{aligned}
D_{\mathrm{HT}}=\frac{1}{64^{2}}( & \frac{Y(1,1)^{2}+Y(1,3)^{2}+Y(3,1)^{2}+Y(3,3)^{2}}{16} \\
& +\frac{Y(2,2)^{2}+Y(2,4)^{2}+Y(4,2)^{2}+Y(4,4)^{2}}{100} \\
& +\frac{Y(1,2)^{2}+Y(1,4)^{2}+Y(2,1)^{2}+Y(4,1)^{2}}{40} \\
& \left.+\frac{Y(2,3)^{2}+Y(3,2)^{2}+Y(3,4)^{2}+Y(4,3)^{2}}{40}\right) .
\end{aligned}
$$

Compared to the pixel-domain distortion calculation in (6), the additional computations include computing $Y$, specifically $64 * E$ and $\widetilde{E}(m) \otimes W I_{1}, 1$ shift (/16), and 2 integer divisions. In computing $Y, 64 * E$ needs 16 shifts, but it only needs to be precomputed once for all modes to be evaluated. Computing $\widetilde{E}(m) \otimes W I_{1}$ requires 16 integer multiplications. Overall, the additional operations at most include 16 multiplications, 2 divisions, and 17 shifts, for a total of 35 operations.

On the other hand, to calculate the distortion using the pixel-domain method according to (6), inverse transform and reconstruction are necessary to reconstruct $\tilde{s}$. The inverse transform needs 64 additions and 16 shifts [13] and the reconstruction needs 16 additions (subtractions). Therefore, the additional operations compared to (6) are 80 additions and 16 shifts, for a total of 96 operations.

From the above analysis, it is apparent that the proposed transform-domain distortion calculation is more efficient than the traditional pixel-domain approach. It should also be noted that the proposed mode decision architecture has additional advantages as explained in Section 3.2.

\section{FAST MODE DECISION ALGORITHMS}

\subsection{Ranking-based mode decision}

For optimal coding performance, the H.264 coder utilizes Lagrange coder control to optimize mode decisions in the rate-distortion sense. When lower complexity is desired, the SATD cost in (9) is used, which requires much simpler computation. Using the SATD cost reduces coding performance since the cost function is only an approximation of the actual RD cost given by (7). In this subsection, we propose a fast intra mode decision algorithm that is based on the following observation: although choosing the mode with the smallest SATD value often misses the best mode in the RD sense, the best mode usually contains smaller SATD cost. In other words, the mode rankings according to the two cost functions are highly correlated.

The basic idea is to rank all candidate modes using the less complex SATD cost, and then evaluate Lagrange RD costs only for the few best modes decided by the ranking. Based on the input HT coefficients of prediction residual signal, the algorithm is described in the following.
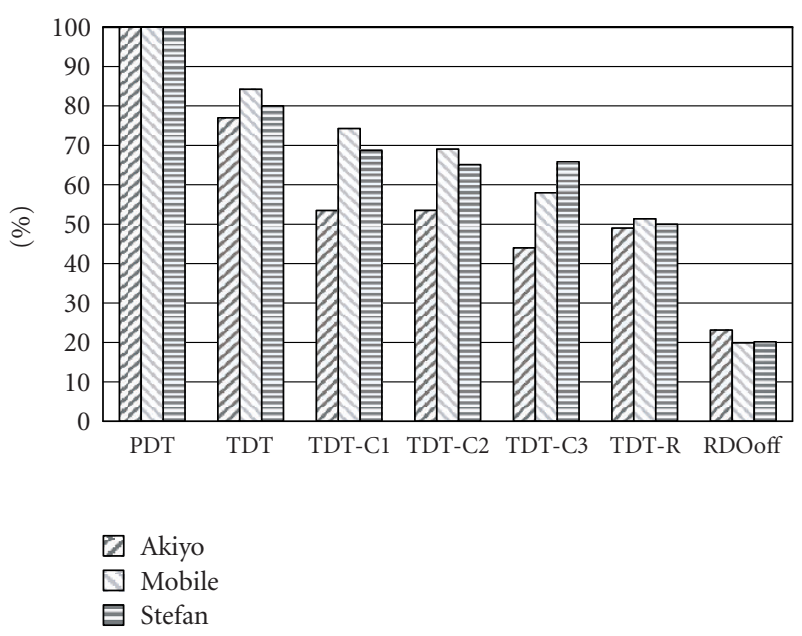

FIGURe 10: Complexity of proposed transcoders (\%) relative to PDT. The threshold values used for Akiyo for TDT-C1, TDT-C2, TDT-C3 are 512, 1024 and 2048 respectively, and for mobile and Stefan, they are 12228, 16834, 24576, and 4096, 12228, 16384, respectively.

First, we compute the HT domain $c_{1}$ for all candidate modes based on normalized HT-domain residual coefficients:

$c_{1}(m)=\left\|(S-\tilde{S}(m)) \otimes W_{2}\right\|_{1}+\lambda_{M} * 4 *\left(1-\delta\left(m=m^{*}\right)\right)$.

Then, we sort the modes according to $c_{1}$ in ascending order, putting the first $k$ smallest modes in the test set $T$. Next, we add DC_Pred into $T$ if it is not in $T$ already. For the modes in $T$, compute

$$
c_{2}(m)=\left\|\left(E-\tilde{E} \otimes W_{1}\right) \otimes W_{2}\right\|_{2}^{2}+\lambda_{M} * R(m) .
$$

We finally select the best mode according to $c_{2}(m)$.

Note that in calculating (9), instead of using Hadamard transform, the distortion SATD is defined as the SAD of HT coefficients since they are already available in the transform-domain transcoder. The parameter $k$ controls the complexity-quality tradeoff. To verify the correlations between rankings using $c_{1}$ and $c_{2}$, a simple experiment is performed. We collect the two costs for all luma $4 \times 4$ blocks in the first frame of all CIF test sequences (see next section) coded with $\mathrm{QP}=28$, and then count the percentage of times when the best mode according to $c_{2}$ is in the test set $T$. This is called the mode prediction accuracy. The results are plotted in Figure 8 as $k$ versus accuracy. The strong correlation between the two costs is evident in the high accuracies shown. In this work, $k$ is set to be 3 .

\subsection{Exploiting temporal correlation}

It is well known that strong correlations exist between adjacent pictures, and it is reasonable to assume that the optimal mode decision results of collocated macroblocks in two adjacent pictures are also strongly correlated. In our earlier work 
TABLE 1: RD performance comparisons with $\mathrm{QP}=27$. Bitrate: kbps, PSNR: dB.

\begin{tabular}{l|c|c|c|c|c}
\hline & & Akiyo & Foreman & Container & Stefan \\
\hline \multirow{2}{*}{ PDT } & Bitrate & 1253.2 & 1695.48 & 2213.6 & 3807.4 \\
& PSNR & 40.40 & 37.30 & 36.24 & 34.63 \\
\hline \multirow{2}{*}{ TDT } & Bitrate & 1577.4 & 2229.1 & 2905.1 & 4812.1 \\
& PSNR & 40.38 & 37.28 & 36.21 & 34.63 \\
\hline \multirow{2}{*}{ TDT-R } & Bitrate & 1579.1 & 2233.3 & 2907.9 & 4809.2 \\
& PSNR & 40.35 & 37.27 & 36.18 & 34.57 \\
\hline
\end{tabular}

TABLE 2: RD performance comparisons with $\mathrm{QP}=30$. Bitrate: kbps, PSNR: dB.

\begin{tabular}{l|c|c|c|c|c}
\hline & & Akiyo & Foreman & Container & Stefan \\
\hline \multirow{2}{*}{ PDT } & Bitrate & 1253.2 & 1659.48 & 2213.6 & 3807.4 \\
& PSNR & 38.63 & 35.84 & 34.77 & 33.26 \\
\hline \multirow{2}{*}{ TDT } & Bitrate & 1258.04 & 1654.16 & 2207.19 & 3795.8 \\
& PSNR & 38.59 & 35.83 & 34.75 & 33.25 \\
\hline \multirow{2}{*}{ TDT-R } & Bitrate & 1257.72 & 1656.48 & 2208.46 & 3789.8 \\
& PSNR & 38.59 & 35.82 & 34.72 & 33.19 \\
\hline
\end{tabular}

[14], we proposed a fast mode decision algorithm for intraonly encoding that exploits the temporal correlation in mode decisions of adjacent pictures. In this subsection, we present the corresponding algorithm that could be within the context of the transform-domain transcoding architecture.

One key step to exploit temporal correlations of macroblock modes is to first measure the difference between the current macroblock and its collocated macroblock in the previously coded picture. If they are close enough, the current macroblock will reuse the mode decision of its collocated macroblock and the entire mode decision process is skipped. In our earlier work, we measured the degree of correlation between two macroblocks in the pixel domain according to a difference measure that accounted not only for the differences between collocated macroblocks, but also the pixels used for intra-prediction of that macroblock. This pixel-domain distance measure may not be applied in the transform-domain architecture since we do not have access to pixel values. We propose to use a distance measure calculated in the transform domain as follows to measure the temporal correlation:

$$
D=\left|S-S_{\text {col }}\right|_{1},
$$

where $S$ is the HT coefficients of current macroblock, and $S_{\text {col }}$ is the HT coefficients of the collocated macroblock. Note that we did not try to include the pixels outside of current macroblock that may be used for intra-prediction. These pixels are difficult to include in the transform-domain distance measure. However, our simulations results show that the exclusion of these pixels did not cause noticeable performance penalty. for all $\mathrm{MBs}$ in picture do

Compute $D$ between the current $\mathrm{MB}$ and associated $\mathrm{MB}$ stored in the buffer based on (26)

if $D>T H$ then

Perform mode decision for the current MB

Update buffer with current MB data

else

Reuse mode decision of the collocated MB in the previous picture

end if

end for

Algorithm 1: Mode decision based on temporal correlation.

The next important element of the proposed algorithm is to prevent accumulation of the distortion resulting from mode reuse. This requires an additional buffer that is updated with coefficients of the current input macroblock only when there is a new mode decision. This strategy allows for differences to be measured based on the original macroblock that was used to determine a particular encoding mode. If the differences were taken with respect to the immediately previous frame, then it would become possible that small differences, that is, less than the threshold, over time would not be detected. In that case, an encoding mode would continue to be reused even though the macroblock characteristics over time have changed significantly.

Figure 9 shows the buffer updating process for several frames containing four macroblocks each. For Frame 0, the mode decisions for all four macroblocks are newly determined and denoted with an $N$. The macroblock data from frame $0\left\{M B_{0}(0,0), M B_{0}(0,1), M B_{0}(1,0), M B_{0}(1,1)\right\}$ are then stored in the frame buffer. For Frame 1, the mode decision has determined that the encoding modes for macroblocks $(0,0)$ and $(0,1)$ will be reused, which are denoted with an $R$, while the encoding modes for macroblocks $(1,0)$ and $(1,1)$ are newly determined. As a result, the buffer is updated with the corresponding macroblock data from frame 1 $\left\{M B_{1}(1,0), M B_{1}(1,1)\right\}$; data for other macroblocks remain unchanged. For Frame 2, only macroblock $(0,1)$ has been newly determined, therefore the only update to the frame buffer is $\left\{M B_{2}(0,1)\right\}$.

It is evident from the above example that the buffer is composed of a mix of macroblock data from different frames. The source of the data for each macroblock represents the frame at which the encoding mode decision was been determined. The data in the buffer is used as a reference to determine whether the current input macroblock is sufficiently correlated and whether the macroblock encoding mode could be reused.

The complete algorithm is given in Algorithm 1.

The threshold $\mathrm{TH}$ can be used to control the qualitycomplexity tradeoff. A larger $T H$ leads to lower quality, but faster mode decision and hence lower computational complexity. 


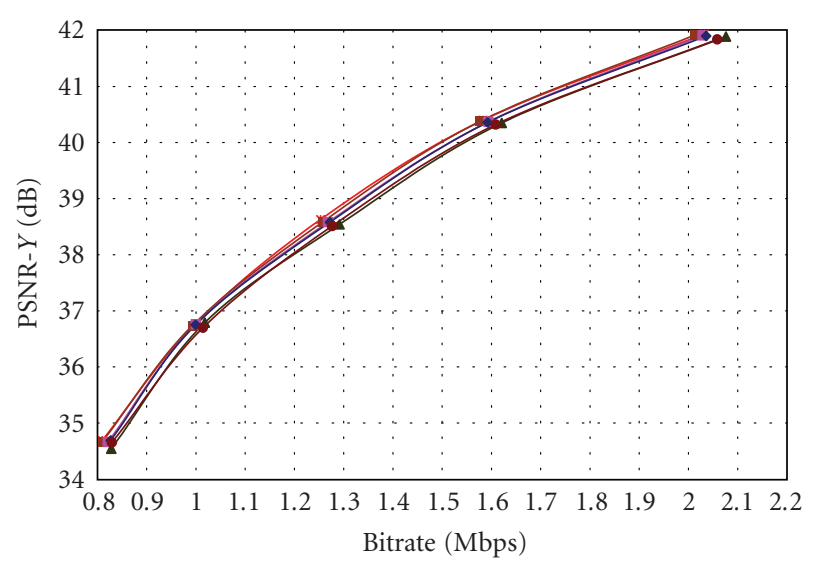

$\begin{array}{ll}\because \text { PDT } & \rightarrow \text { TDT-C(1024) } \\ \because \text { TDT } & \rightarrow \text { TDT-C(2048) } \\ - \text { TDT-C(512) } & \rightarrow \text { PDT-RDOoff }\end{array}$

(a)

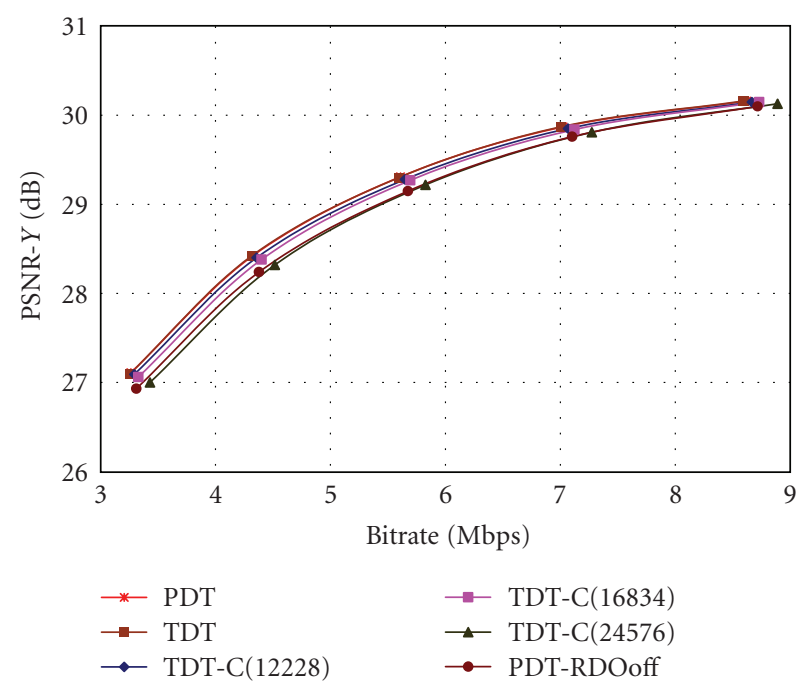

(b)

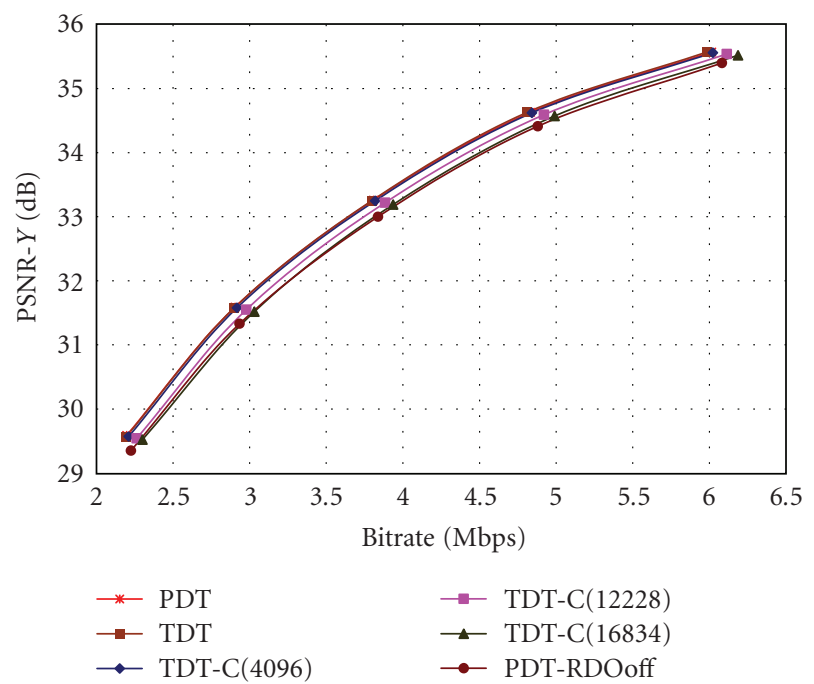

(c)

FIGURE 11: RD performance evaluation of TDT-C transcoder with different thresholds: (a) Akiyo; (b) Mobile; (c) Stefan.
TABLE 3: RD performance comparisons with $\mathrm{QP}=33$. Bitrate: kbps, PSNR: dB.

\begin{tabular}{l|c|c|c|c|c}
\hline & & Akiyo & Foreman & Container & Stefan \\
\hline \multirow{2}{*}{ PDT } & Bitrate & 993.2 & 1249.0 & 1673.9 & 2904.8 \\
& PSNR & 36.72 & 34.29 & 33.19 & 31.59 \\
\hline \multirow{2}{*}{ TDT } & Bitrate & 993.2 & 1246.6 & 1671.3 & 2899.5 \\
& PSNR & 36.74 & 34.27 & 33.18 & 31.58 \\
\hline \multirow{2}{*}{ TDT-R } & Bitrate & 993.9 & 1248.0 & 1673.4 & 2896.5 \\
& PSNR & 36.73 & 34.27 & 33.16 & 31.52 \\
\hline
\end{tabular}

\section{SIMULATION RESULTS}

In this section, we report results to demonstrate the effectiveness of the proposed architectures and algorithms. We compare the coding efficiency and complexity of the pixel-domain transcoder (PDT) to the transform-domain transcoder (TDT) with RDO turned on. The PDT, as shown in Figure 1, uses conventional coefficient conversion method and conventional mode decision algorithm. Chen's fast IDCT implementation [10] is used in MPEG-2 decoding. In TDT, as shown in Figure 2, the proposed transcoding architecture, integer DCT-to-HT conversion (Section 2.3), and transform-domain mode decision (Section 3 ) are implemented. We also evaluate the performance of the proposed fast mode decision algorithms (Section 4) within the context of the TDT architecture, namely the fast mode decision based on ranking (TDT-R) and the algorithm based on temporal correlation (TDT-C). Comparisons are made to the PDT architecture with RDO on and off.

The experiments are conducted using 100 frames of standard test sequences at CIF resolution. The sequences are all intra-encoded at a frame rate of $30 \mathrm{~Hz}$ and bit-rate of $6 \mathrm{Mbps}$ using the public domain MPEG-2 software [15]. The resulting bitstreams are then transcoded using the various architectures. The transcoders are implemented based on MSSG MPEG-2 software codec and H.264 JM7.6 reference code [16].

Tables 1-3 summarize the RD performance of the reference transcoder, that is, PDT, and proposed transcoders, TDT and TDT-R, while Figure 10 shows the complexity results for two sequences and QP 30. Results are similar for other sequences and QP values. It is noted that the complexity is measured by the CPU time consumed by transcoders. All simulations are performed on a PC running Windows XP with an Intel Pentium-4 CPU $2.4 \mathrm{GHz}$. The software is compiled with the Intel C++ Compiler v7.0. Several key observations regarding the results are discussed below.

The first notable point is that the TDT architecture achieves virtually the same RD performance as PDT. Also, the computational savings of TDT over PDT are typically around $20 \%$. These savings come partly from the reduced complexity achieved by the S-transform compared to pixel-based conversion and partly from the reduced complexity in the mode 
decision process. Recall that both architectures employ RDO, but the mode decision in the transform-domain architecture performs the distortion calculation in the transformdomain, thereby eliminating certain operations.

If we further analyze the performance of TDT-R, we observe that this algorithm saves approximately $50 \%$ of the computation compared to the PDT architecture. Compared to the TDT architecture with full RDO, up to $30 \%$ savings are achieved. Furthermore, these computational savings are achieved with negligible degradation in PSNR. The results show less than $0.1 \mathrm{~dB}$ loss for all test cases.

The simulation results using various threshold values for transcoder TDT-C are shown in Figure 11. In this figure, each RD curve is generated using 5 different QP values: 24, 27, 30, 33, and 36. As references, we plot PDT with RDO on and off, as well as TDT. The complexity comparison is shown in Figure 10. It can be seen that different threshold values provide different tradeoffs of RD performance and computational complexities. Relative to TDT, up to $40 \%$ computation can be saved with less than $0.2 \mathrm{~dB}$ loss in quality. These results show that turning off RDO provides the lowest complexity, but also results in lower quality. For the three sequences shown in the figure, the quality degradation is in the range of $0.2-0.4 \mathrm{~dB}$. The benefits of the TDT-C scheme is that it offers flexible tradeoffs between coding efficiency and complexity.

Both TDT-R and TDT-C provide significant saving in complexity relative to exhaustive RDO algorithm. It appears though that TDT-R is a more effective approach since it incurs almost no loss in RD performance and achieves comparable complexity reduction to TDT-C. Perhaps a combination of the two approaches would yield further complexity reduction and better tradeoff.

\section{CONCLUSIONS}

We proposed an efficient transform-domain MPEG-2 to H.264 intra-video transcoder. The transform-domain architecture is equivalent to the conventional pixel-domain implementation in terms of functionality, but it has significantly lower complexity with no loss in coding efficiency. We achieved complexity reduction with a transform-domain architecture that utilizes a direct DCT-to-HT coefficient conversion and a transform-domain mode decision. The transform-mode decision is enabled by calculating distortion based on transform coefficients. We also presented two fast mode decision algorithms that are able to operate within the context of the transform-domain architecture. Both of these algorithms demonstrated that further reductions in complexity with negligible loss in quality could be achieved.

\section{REFERENCES}

[1] "ITU-T Rec. H.264-ISO/IEC 14496-10: Advanced Video Coding," 2003.

[2] Z. Zhou, S. Sun, S. Lei, and M.-T. Sun, "Motion information and coding mode reuse for MPEG-2 to H.264 transcoding," in Proceedings of IEEE International Symposium on Circuits and Systems (ISCAS '05), vol. 2, pp. 1230-1233, Kobe, Japan, May 2005.
[3] X. Lu, A. M. Tourapis, P. Yin, and J. Boyce, "Fast mode decision and motion estimation for H.264 with a focus on MPEG2/H.264 transcoding," in Proceedings of IEEE International Symposium on Circuits and Systems (ISCAS '05), vol. 2, pp. 1246-1249, Kobe, Japan, May 2005.

[4] T. Qian, J. Sun, D. Li, X. Yang, and J. Wang, "Transform domain transcoding from MPEG-2 to H.264 with interpolation drift-error compensation," IEEE Transactions on Circuits and Systems for Video Technology, vol. 16, no. 4, pp. 523-534, 2006.

[5] K. B. Bruce, L. Cardelli, and B. C. Pierce, "Comparing object encodings," in Proceedings of 3rd International Symposium on Theoretical Aspects of Computer Software (TACS '97), M. Abadi and T. Ito, Eds., vol. 1281 of Lecture Notes in Computer Science, pp. 415-438, Springer, Sendai, Japan, September 1997.

[6] H. Yu, “Joint 4:4:4 Video Model (JFVM) 2," JVT-R205, 2006.

[7] J. Xin, A. Vetro, and H. Sun, "Converting DCT coefficients to H.264/AVC transform coefficients," in Proceedings of IEEE Pacific-Rim Conference on Multimedia (PCM '04), vol. 2, pp. 939-946, Tokyo, Japan, November 2004.

[8] B. Shen, "From 8-tap DCT to 4-tap integer-transform for MPEG to H.264/AVC transcoding," in Proceedings of the International Conference on Image Processing (ICIP '04), vol. 1, pp. 115-118, Singapore, October 2004.

[9] C. Y. Park and N. I. Cho, "A fast algorithm for the conversion of DCT coefficients to H.264 transform coefficients," in Proceedings of the International Conference on Image Processing (ICIP '05), vol. 3, pp. 664-667, Genova, Italy, September 2005.

[10] W.-H Chen, C. Smith, and S. Fralick, "A fast computation algorithm for the discrete cosine transform," IEEE Transactions on Communications, vol. 25, no. 9, pp. 1004-1009, 1977.

[11] H. S. Malvar, A. Hallapuro, M. Karczewicz, and L. Kerofsky, "Low-complexity transform and quantization in H.264/AVC," IEEE Transactions on Circuits and Systems for Video Technology, vol. 13, no. 7, pp. 598-603, 2003.

[12] J. Xin, A. Vetro, and H. Sun, "Efficient macroblock codingmode decision for H.264/AVC video coding," in Proceedings of the 24th Picture Coding Symposium (PCS '04), pp. 53-58, San Francisco, Calif, USA, December 2004.

[13] A. Hallapuro, M. Karczewicz, and H. Malvar, "Low complexity transform and quantization-part II: extensions," JVT-B039, 2002.

[14] J. Xin and A. Vetro, "Fast mode decision for intra-only H.264/AVC coding," in Proceedings of the 25th Picture Coding Symposium (PCS '06), Beijing, China, April 2006.

[15] "MPEG-2 encoder/decoder v1.2," 1996, by MPEG Software Simulation Group, http://www.mpeg.org/MPEG/MSSG/.

[16] "H.264/AVC reference software JM7.6," 2003, http://iphome .hhi.de/suehring/tml/download/.

Jun Xin received the B.E. degree from Southeast University, Nanjing, China, in 1993, the M.E. degree from Institute of Automation, Chinese Academy of Sciences, Beijing, China, in 1996, and the Ph.D. degree from University of Washington, Seattle, Wash, USA in 2002, all in electrical engineering. Since August 2003, he has been with Mitsubishi Electric Research Laboratories (MERL), Cambridge, Mass, USA. From 1996 to 1998, he was a Software Engineer at Motorola-ICT Joint R\&D Lab, Beijing, China. His research interests include digital video compression and communication. He has been an IEEE Member since 2003. 
Anthony Vetro received the B.S., M.S., and Ph.D. degrees in electrical engineering from Polytechnic University, Brooklyn, NY. He joined Mitsubishi Electric Research Labs, Cambridge, Mass, USA, in 1996, where he is currently a Senior Team Leader and responsible for research related to the encoding, transport, and consumption of multimedia content. He has published more than

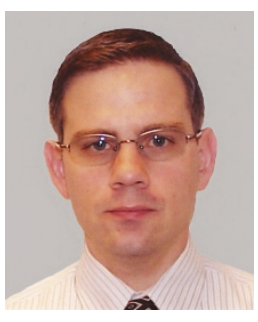
100 papers and has been an active member of the MPEG and JVT Standardization Committee for several years. $\mathrm{He}$ is currently serving as an Editor for multiview video coding amendment of H.264/AVC. Dr. Vetro serves on the program committee for various conferences and has held several editorial positions. He is currently an Associate Editor for IEEE Signal Processing Magazine and Chair-Elect of the Technical Committee on Multimedia Signal Processing of the IEEE Signal Processing Society, as well as the Technical Committees on Visual Signal Processing and Communications and Multimedia Systems and Applications of the IEEE Circuits and Systems Society. He recently served as a Conference Chair for ICCE 2006 and a Tutorials Chair for ICME 2006, and has been a Member of the Publications Committee of the IEEE Transactions on Consumer Electronics since 2002. Dr. Vetro has also received several awards for his work on transcoding, including the 2003 IEEE Circuits and Systems CSVT Transactions Best Paper Award and the 2002 Chester Sall Award. He is a Senior Member of the IEEE.

Huifang Sun graduated from Harbin Engineering Institute, Harbin, China, and received the Ph.D. degree from University of Ottawa, Canada. He joined Electrical Engineering Department of Fairleigh Dickinson University as an Assistant Professor in 1986 and was promoted to an Associate Professor before moving to Sarnoff Corporation in 1990. He joined Sarnoff Lab as a member of technical staff and was promoted to a

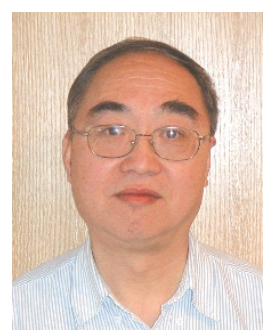
Technology Leader of Digital Video Communication later. In 1995, he joined Mitsubishi Electric Research Laboratories (MERL) as Senior Principal Technical Staff and was promoted as Vice President and Fellow of MERL and Deputy Director of Technology Lab in 2003. His research interests include digital video/image compression and digital communication. He has coauthored two books and more than 130 journal/conference papers. He holds 43 US patents and has more pending. He received Technical Achievement Award for optimization and specification of the Grand Alliance HDTV video compression algorithm in 1994 at Sarnoff Lab. He received the Best Paper Award of 1992 IEEE, Transaction on Consumer Electronics, the Best Paper Award of 1996 ICCE, and the Best Paper Award of 2003 IEEE Transaction on CSVT. He is now an Associate Editor for IEEE Transaction on Circuits and Systems for Video Technology and was the Chair of Visual Processing Technical Committee of IEEE Circuits and System Society. He is an IEEE Fellow.

Yeping Su was born in Rugao, Jiangsu, China. He received his Ph.D. degree (2005) in electrical engineering at the University of Washington, Seattle. He interned for EnGenius Technologies at Bellevue, Wash, USA (2001), Microsoft Research at Redmond, Wash, USA (2003), and Mitsubishi Electric Research Labs at Cambridge, Mass, USA (2004). He was a member of technical staff at Thomson Corporate Research Lab

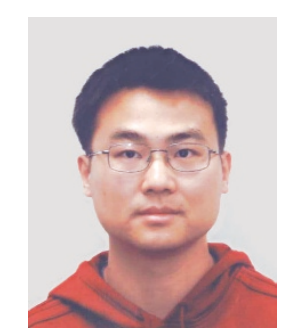

at Princeton, NJ, from 2005 to 2006. He is currently a member of technical staff at Sharp Labs of America at Camas, Wash. 